\title{
High-Accuracy AM-FM Radar with an Active Reflector
}

\author{
Mun Gak Choi, ${ }^{1}$ Dong Sik Woo, ${ }^{2}$ Hyun Chul Choi, ${ }^{1}$ and Kang Wook Kim ${ }^{1}$ \\ ${ }^{1}$ School of Electronics Engineering, Kyungpook National University, 80 Daehak-ro, Buk-gu, Daegu 41566, Republic of Korea \\ ${ }^{2}$ Department of Aviation Information and Communications Engineering (KAI-TECH), Kyungwoon University, \\ 730 Gangdong-ro, Sandong-myun, Gumi 39610, Republic of Korea \\ Correspondence should be addressed to Kang Wook Kim; kang_kim@ee.knu.ac.kr
}

Received 3 August 2016; Revised 27 November 2016; Accepted 9 January 2017; Published 30 January 2017

Academic Editor: Eduard Llobet

Copyright (C) 2017 Mun Gak Choi et al. This is an open access article distributed under the Creative Commons Attribution License, which permits unrestricted use, distribution, and reproduction in any medium, provided the original work is properly cited.

An amplitude-modulated and frequency-modulated (AM-FM) radar with an active reflector to produce high-accuracy distance measurements is proposed and demonstrated in this paper. The proposed radar consists of an AM-FM base module and an active reflector. The combination of AM and FM modulations resolves ambiguity of the absolute distance in typical AM radars, while improving range accuracy in typical FM radars with narrow bandwidth. Also, the active reflector, which translates the frequency of the received signal, resolves the problem of phase detection interference due to the direct Tx-to-Rx leakage in AM radars. In this paper, the operating principle, experimental tests, and analysis are presented. The implemented AM-FM radar operates in X-band (Tx: $10.5 \mathrm{GHz}, \mathrm{Rx}: 8.5 \mathrm{GHz}$ ) with the $620 \mathrm{MHz}$ bandwidth. The measured range accuracy of less than $\pm 10 \mathrm{~mm}$ at a distance of $70 \mathrm{~m}$ is obtained.

\section{Introduction}

Accurate noncontact distance measurement systems are widely used in various applications. Position measurement methods can be divided into different categories depending on the utilized technologies such as ultrasound, GPS, laser, and RF/microwave waves [1]. Attempts to use optical fibers for the detection of cracks and vibrations in bridges or tunnels have also been reported [2].

These distance measurement technologies have their own advantages and disadvantages. An ultrasonic system is limited to short-range distances in a small area because of its low precisions; it is widely employed in rear bumper proximity sensors of commercial vehicles. The accuracy of typical GPSbased distance measuring systems is approximately $10 \mathrm{~m}$, and, therefore, it is not suitable for the detection of structural faults. Laser sensors have a range of up to several hundred meters and mm-level accuracy. However, their performance degrades rapidly under the influence of heavy raining or foggy weather and with lens or reflector contamination. An alignment of the laser beam is also required for accurate measurements. On the other hand, a radar-based sensor, using electromagnetic radiation, can operate reliably under harsh weather conditions. Therefore, the radar technology is widely used not only in military systems but also in a number of industrial applications such as automotive radars, blind spot monitoring, and level gauges. Active researches have been performed for the high-resolution radar technology with the mm-level accuracy [3-9]. Both ultrawideband (UWB) [3-7] and wideband frequency-modulated continuous-wave (FMCW) $[8,9]$ techniques were used to achieve this level of precision.

In this paper, we propose a practical ranging system with mm-accuracy using AM-FM radar combined with the active reflectors. The frequency translating active reflector is utilized in order to significantly reduce the phase detection ambiguity in AM radars related to interference or multipath signal reception. The proposed AM-FM radar uses narrower bandwidth as compared with other methods.

\section{AM-FM Radar and the Active Reflector}

2.1. Overview of the Proposed AM-FM Radar. The proposed AM-FM radar module combines amplitude modulation (AM) and frequency modulation (FM) based radars with an active reflector. A simplified block diagram of the proposed system is shown in Figure 1. The proposed system consists 


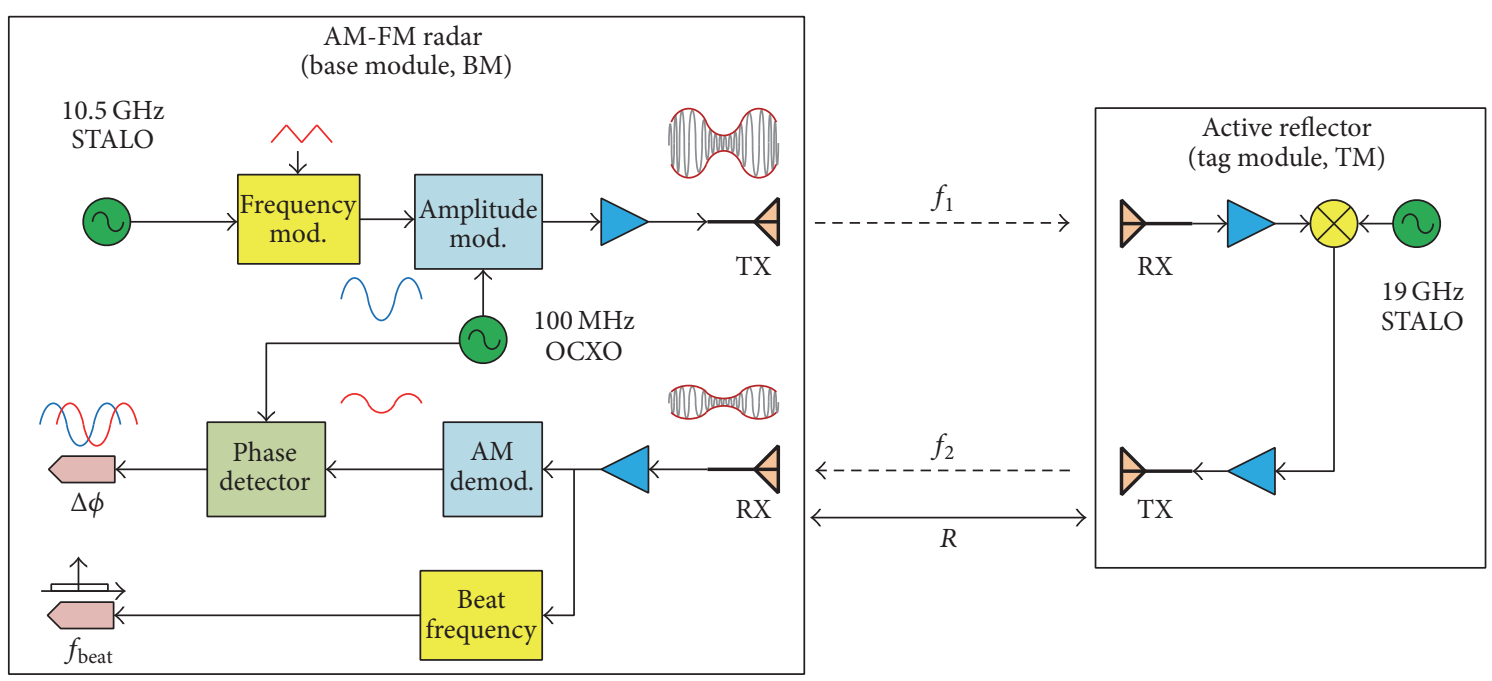

FIgURE 1: Simplified block diagram of the proposed AM-FM radar.

of two parts: an AM-FM radar module (base module, BM) and an active reflector module (tag module, TM). In the AM-FM radar base module, a CW microwave signal is first frequency-modulated and then amplitude-modulated. The AM-FM signal is transmitted toward the active reflector. The active reflector at the target location receives the AMFM signal, translates the center frequency of the signal, and retransmits the signal with amplification. Then, the base radar module receives the retransmitted signal from TM and determines the absolute distance with combination of the beat frequency due to FM modulation and phase delay due to AM modulation. The beat signal due to FM modulation helps to obtain the rough absolute distance on the order of $1 \mathrm{~m}$. The phase delay due to AM modulation helps to resolve the distance with mm-accuracy. The ambiguity of absolute distance in AM radars is overcome with the combination of the FM modulation. Also, with an active reflector, which translates frequency of the received signal and retransmits it toward the base system, the phase detection interference problem in typical AM radars can be resolved.

In the next paragraphs, FMCW and amplitude-modulated continuous-wave (AMCW) radar principles are briefly summarized, and the benefits of adapting active reflectors are described.

2.2. FMCW Radar. A typical bistatic and homodyne FMCW radar configuration $[10,11]$ is adapted for the proposed AMFM radar system. In general radar system, range accuracy is a concept that should be distinguished from range resolution but is related to it. The range resolution $(\delta R)$ and bandwidth $(B)$ relation is

$$
\delta R=\frac{c}{2 B}
$$

where $c$ is speed of light. High range resolution increases range accuracy [12]. Therefore, to achieve high accuracy in radar system, wide bandwidth is essentially needed.
On the other hand, another limitation is present due to the signal processing. Typically, in order to acquire the beat frequency of the received signal, an analog beat signal is first sampled using an analog-to-digital converter, and then the fast Fourier transform (FFT) algorithm is utilized. As the result of FFT, the beat frequency is discretely distributed. The maximum beat frequency error equals the FFT step size, and the differences exist between the real beat frequency and the discrete beat frequency by FFT [13]. The FFT step size $(\Delta f)$ can be calculated as follows:

$$
\Delta f=\frac{f_{s}}{N_{s}}
$$

where $f_{s}$ is the sampling frequency and $N_{s}$ is the total number of data samples during the sampling period. Thus, the range step size $\left(\Delta R_{\max }^{\mathrm{FFT}}\right)$ according to the FFT step size is as follows:

$$
\Delta R_{\max }^{\mathrm{FFT}}=\frac{c T \Delta f}{2 B}=\frac{c T}{2 B} \cdot \frac{f_{s}}{N_{s}},
$$

where $T$ is chirp period. As shown in (1) and (3), high range accuracy requires both wide frequency bandwidth and highperformance hardware.

2.3. AMCW Radar. The AMCW radar system was reported in [14], and certain drawbacks of the AMCW radar were pointed out. First, the maximum measurable distance, without ambiguity, is limited to the wavelength of the modulation frequency. Second, the leakage power between transmitter $(\mathrm{Tx})$ and receiver $(\mathrm{Rx})$ antennas induces the phase detection error. In spite of these problems, the AMCW radar has several advantages: (1) a very simple structure and (2) high range accuracy achievable by an increase in the modulation frequency. Consequently, the AMCW method was used in some special applications such as a short-range radar and plasma diagnostics [15]. 


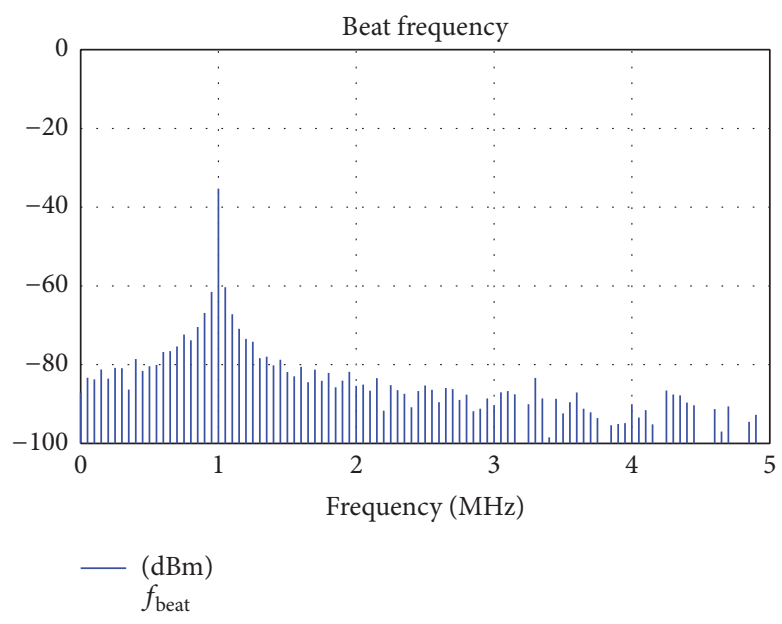

(a) FFT result of the beat signal

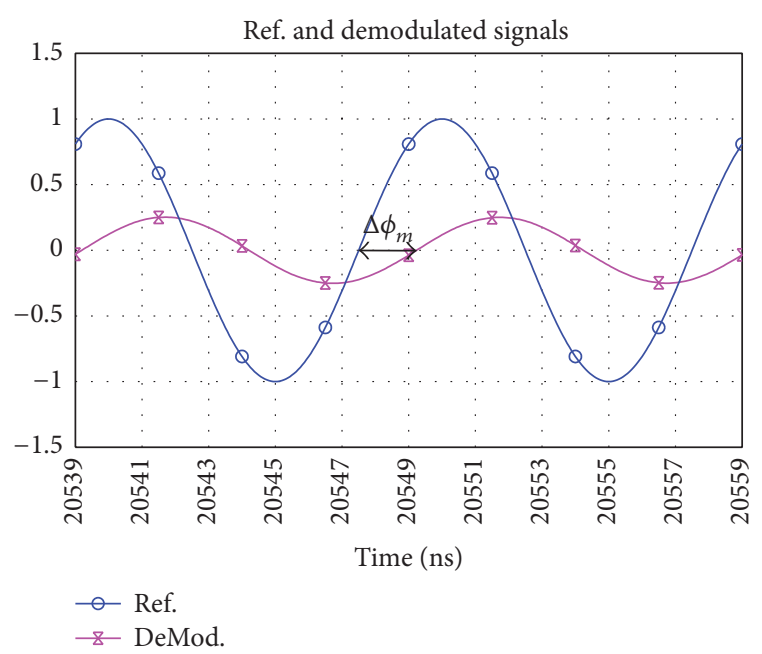

(b) Phase difference

Figure 2: Typical cases of beat frequency and phase difference.

TABLE 1: Range errors in the AMCW radar with no leakages.

\begin{tabular}{lccc}
\hline $\begin{array}{l}\text { Phase detection } \\
\text { error }\end{array}$ & $f_{m}=10 \mathrm{MHz}$ & $f_{m}=50 \mathrm{MHz}$ & $f_{m}=100 \mathrm{MHz}$ \\
\hline $1^{\circ}$ & $41.67 \mathrm{~mm}$ & $8.33 \mathrm{~mm}$ & $4.17 \mathrm{~mm}$ \\
$5^{\circ}$ & $208.33 \mathrm{~mm}$ & $41.67 \mathrm{~mm}$ & $20.83 \mathrm{~mm}$ \\
$10^{\circ}$ & $416.67 \mathrm{~mm}$ & $83.33 \mathrm{~mm}$ & $41.67 \mathrm{~mm}$ \\
\hline
\end{tabular}

The transmitted signal of the AMCW radar is amplitudemodulated, consisting of the carrier $\left(f_{c}\right)$ and double sidebands $\left(f_{c} \pm f_{m}\right)$ in the frequency domain. The distance $R_{\mathrm{AM}}$ between the radar and the target is

$$
R_{\mathrm{AM}}=\frac{c \cdot \Delta \phi_{m}}{4 \pi f_{m}}
$$

where $\Delta \phi_{m}$ is the phase difference between the reference and the received signals and $f_{m}$ is the modulation frequency. Table 1 summarizes the range errors corresponding to the different phase detection errors and $f_{m}$. Here, the ambiguity of absolute distance exists depending on the modulation frequency. Distance ambiguity of AM radar with $100 \mathrm{MHz}$ modulation frequency is $1.5 \mathrm{~m}$.

In a typical AMCW radar, a portion of the transmitted signal is routed to the received signal path through $\mathrm{Tx} / \mathrm{Rx}$ antennas or isolators. The major factor causing an error in the AMCW radar is the ratio of the main echo signal and the direct Tx-to-Rx leakage signal. For $\pm 1^{\circ}$ phase accuracy, the echo signal should be at least $12 \mathrm{~dB}$ stronger than the direct leakage signal. However, the direct leakage power is kept constant, while the echo signal decreases as the distance $R$ increases. In the proposed AM-RM radar, a frequencyshifting active reflector removes the problem of the direct Txto- $\mathrm{Rx}$ signal leakage.

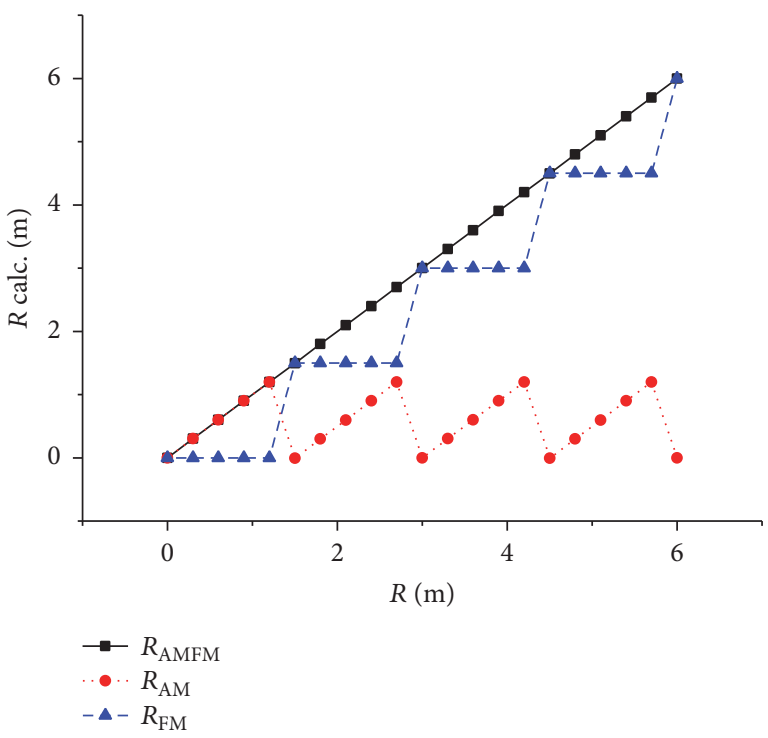

FIgURE 3: Distance estimation of AMCW (red), FMCW (blue), and combination of AM-FM (black) radar.

2.4. Combined AM-FM Radar. The absolute distance between the AM-FM radar base module and the active reflector $\left(R_{\mathrm{AMFM}}\right)$ can be calculated as follows:

$$
R_{\mathrm{AMFM}}=R_{\mathrm{AM}}+R_{\mathrm{FM}}=\frac{c \cdot \Delta \phi_{m}}{4 \pi f_{m}}+\frac{c T f_{\text {beat_FFT }}}{2 B},
$$

where $R_{\mathrm{AM}}$ is the range due to $\Delta \phi_{m}$ of the AMCW radar and $R_{\mathrm{FM}}$ is the range due to the FFT result of beat frequency ( $f_{\text {beat_FFT }}$ ) of the FMCW radar. $R_{\mathrm{FM}}$ is discrete due to the FFT step size, but $R_{\mathrm{AM}}$ varies continuously within unambiguous region. Figure 2 presents typical cases of $f_{\text {beat_FFT }}$ and $\Delta \phi_{m}$. The absolute distance estimation by combining the FM and AM distance measurements using (5) is shown graphically in Figure 3. As a result, high-accuracy distance measurement can be achieved. 
2.5. Active Reflector. An active reflector is typically used in the radar system for calibration. The active radar calibrator (ARC) is composed of two antennas (sending and receiving) and an RF amplifier. Despite its small size, it is possible to obtain a high standard radar cross section (RCS) value in the radar system [16]. In the proposed AM-FM radar system, the active reflector translates the frequency of the incoming signal and retransmits it toward the radar base module. Adopting the active reflector has two advantages. First, the problem of the direct Tx-to-Rx leakage through antennas or isolators can be solved due to frequency translation, removing the serious drawback of the AMCW radar system. Second, a high conversion gain can be achieved by splitting the gain between the base module and the active reflector module.

\section{Implementation and Measurement Results}

3.1. Design and Implementation. The proposed AM-FM radar consists of two parts as presented in Figure 1. One is the base module (BM, AM-FM radar) and the other is the tag module (TM, active reflector). In the $\mathrm{BM}$, a $100 \mathrm{MHz}$ OCXO provides the reference signal for the phase detector, as well as the baseband signal of the amplitude modulation. The OCXO is also used as a reference of the PLL in the $10.5 \mathrm{GHz}$ stable local oscillator (STALO) and as a system clock for the direct digital synthesizer (DDS), providing the linear frequency modulation. An amplitude-modulated and frequency-modulated $10.5 \mathrm{GHz}$ signal is transmitted through the Tx antenna of the BM and is received by the Rx antenna of the TM at the target location. The TM converts the center frequency of the received signal from $10.5 \mathrm{GHz}$ to $8.5 \mathrm{GHz}$ with the help of $19 \mathrm{GHz}$ STALO and retransmits the filtered and amplified signal using the Tx of the TM. Then, the BM receives the $8.5 \mathrm{GHz}$ AM-FM signal and demodulates it into the phase delayed signal which is produced by the envelope detector and also into the beat signal with the help of the frequency mixer. The combination of the phase difference and the beat frequency enables calculating the absolute distance of the target with high accuracy. The overall gain budget is calculated using a spreadsheet and verified with the system simulator, NI AWR Microwave Office (MWO).

Figure 4 shows the fabricated RF front-end modules of the AM-FM radar. For the RF circuit, 20 mil thick RO4003 $\left(\varepsilon_{r}=3.38 ; \tan \delta=0.0027\right)$ substrate is used. Passive components such as the filter, power divider, and power detector were simulated using the 2.5 D EM simulator of the NI AWR MWO. RF PCBs are mounted in a metal housing in separate channels. Regulators for the bias and other circuits are mounted on the bottom side of the housing.

The transmit power of the $\mathrm{BM}$ is $+17.6 \mathrm{dBm}$. The FM chirp signal is generated using the DDS. With the FM bandwidth of $420 \mathrm{MHz}$, the chirp period of $200 \mu$ s is achieved for both rising and falling. The AM signal is generated using the $\mathrm{RF}$ mixer and the combiner. The modulation index is $20 \%$. Total RF bandwidth results in $620 \mathrm{MHz}$. The Tx spectrum of the BM is shown in Figure 5(a), and the down-converted spectrum of the TM is shown in Figure 5(b). The IQ-mixer and NI DAQ are used for the phase calculation, while the
TABLE 2: Proposed AM-FM radar specifications.

\begin{tabular}{lcc}
\hline Parameters & Unit & Value \\
\hline Base module & $\mathrm{dBm}$ & \\
Tx power & $\mathrm{GHz}$ & 17.6 \\
Tx frequency & $\mathrm{GHz}$ & 10.5 \\
Rx frequency & $\%$ & 8.5 \\
AM modulation index & $\mathrm{dB}$ & 20 \\
AM conversion gain & $\mathrm{MHz}$ & 3.5 \\
FM bandwidth & $\mathrm{kHz}$ & 420 \\
FM sweep frequency & $\mathrm{dB}$ & 2.5 \\
FM conversion gain & & 61.5 \\
\hline Tag module & $\mathrm{GHz}$ & \\
Rx frequency & $\mathrm{GHz}$ & 8.5 \\
Tx frequency & $\mathrm{dB}$ & 10.5 \\
Conversion gain & & 32 \\
\hline Antenna (horn) & $\mathrm{dBi}$ & 24 \\
Gain & $\circ$ & 20 \\
Beam width & &
\end{tabular}

beat frequency is measured with the aid of the NI-SCOPE. Detailed system specifications are summarized in Table 2.

3.2. Measurements. To verify performance of the proposed system, outdoor experiments were carried out at a University parking lot. The measurement setup is presented in Figure 6. The distance between the $\mathrm{BM}$ and the TM was varied from $71 \mathrm{~m}$ to $73 \mathrm{~m}$. The phase difference and the beat frequency were measured at $10 \mathrm{~cm}$ intervals. For fine measurements, distance intervals of $5 \mathrm{~cm}$ and $1 \mathrm{~cm}$ were used.

The measured phase difference and beat frequency are plotted in Figure 7(a). A commercial data acquisition module (NI USB-5133) and its FFT function software were used to analyze the FM data. With $10 \mathrm{MHz}$ of $f_{s}$ and number of samples of 1024, the FFT step size was $9.766 \mathrm{kHz}$. In the proposed system, the FM bandwidth is $420 \mathrm{MHz}$, and the sweep frequency is $2.5 \mathrm{kHz}$. The distance of phase ambiguity in the AM radar $(100 \mathrm{MHz})$ is $1.5 \mathrm{~m}$, and the corresponding FFT step for $1.5 \mathrm{~m}$ distance is $21 \mathrm{kHz}$. Therefore, there are $\sim 2$ beat frequency hops within a phase change of $2 \pi$ in the AM measurement. The measured beat frequency of the FM signal is divided by $21 \mathrm{kHz}$ and a truncated integer bin number is obtained to calculate the base distance estimated by the FM measurement (i.e., (bin number) $\times 1.5 \mathrm{~m}$ ). Then, the distance calculated from the phase delay of the AM modulation signal is added to obtain the absolute distance using (5) as shown in Figure 7(b).

A zoomed portion of the fine-measured data in Figure 7(b) is plotted in Figure 7(c) with the calculated error. From the figure, the precision of the tested system is estimated as $\pm 10 \mathrm{~mm}$.

3.3. Discussion. Competing technologies for the high-accuracy distance measurements include FMCW and UWB. 


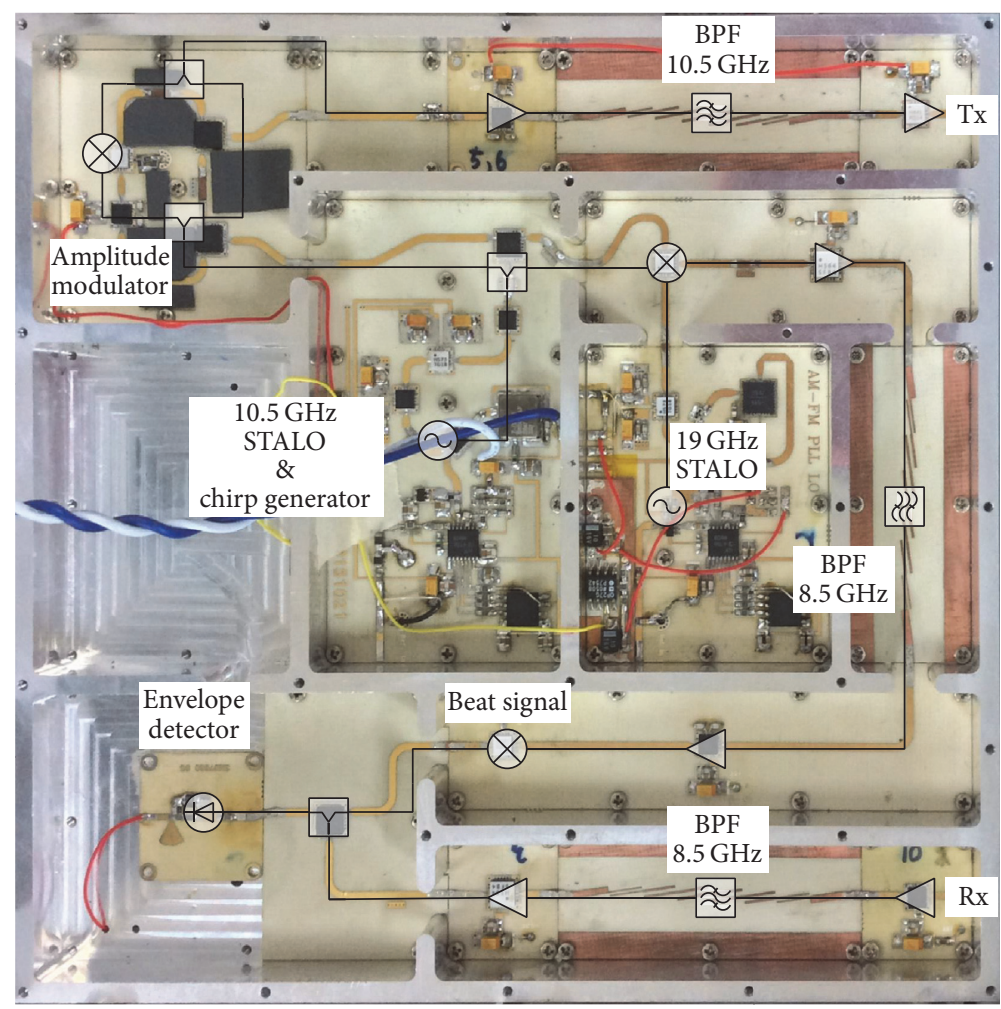

(a) Fabricated base module (BM)

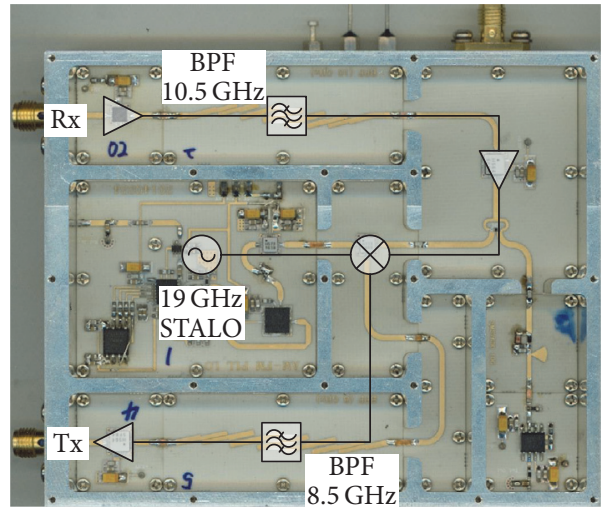

(b) Fabricated tag module (TM)

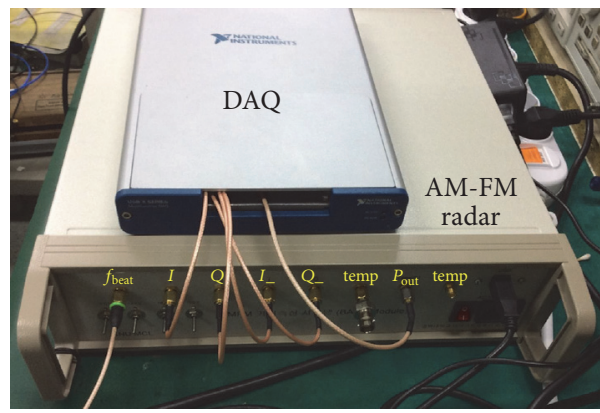

(c) AM-FM radar module (BM)

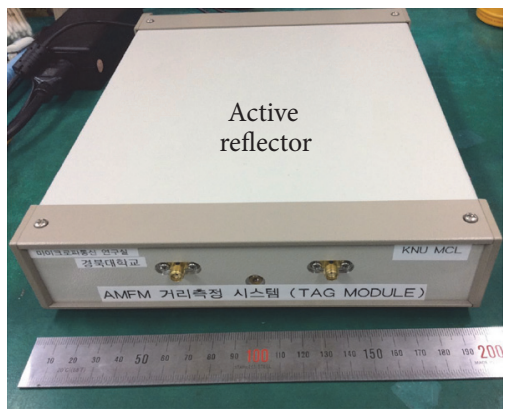

(d) Active reflector module (TM)

Figure 4: Fabricated RF front-end modules ( $a, b)$ and the implemented AM-FM radar (c, d).

These methods use wide frequency bandwidth from 1000 to $5000 \mathrm{MHz}$ with the error level from $5 \mathrm{~mm}$ to $20 \mathrm{~cm}$. Table 3 presents a summary of various distance measurement systems, associated bandwidth, and errors. As can be seen, the proposed system has been demonstrated to achieve high accuracy, in spite of using narrower bandwidth as compared with other systems.

\section{Conclusion}

A high-accuracy distance measuring system combining the $\mathrm{AM}$ and FM radars and the active reflector is proposed. Its operating principle, experimental tests, and analysis are presented. The proposed system operates at center frequencies of $10.5 \mathrm{GHz}(\mathrm{Tx})$ and $8.5 \mathrm{GHz}(\mathrm{Rx})$ and occupies $620 \mathrm{MHz}$ bandwidth around each of the center frequencies. The transmit power was $+17 \mathrm{dBm}$, and standard horn antennas with a gain of $24 \mathrm{dBi}$ were used. Compared with other methods, the proposed system used narrower frequency bandwidth but achieved high range accuracy. The measured distance error with respect to the absolute distance was below $\pm 10 \mathrm{~mm}$. From these results, the proposed AM-FM radar is expected to be applied to various fields that need high-accuracy distance measurements including detection of the vibration or cracks in concrete structures or bridges. Also, accurate distance monitoring for multiple target locations is possible with multiple active reflectors with their corresponding IDs. 


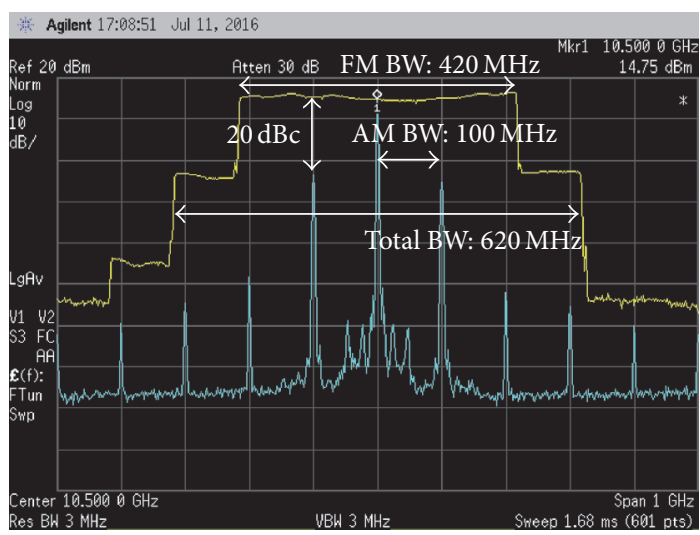

- FM

- AM

(a) Tx spectrum of the base module (BM)

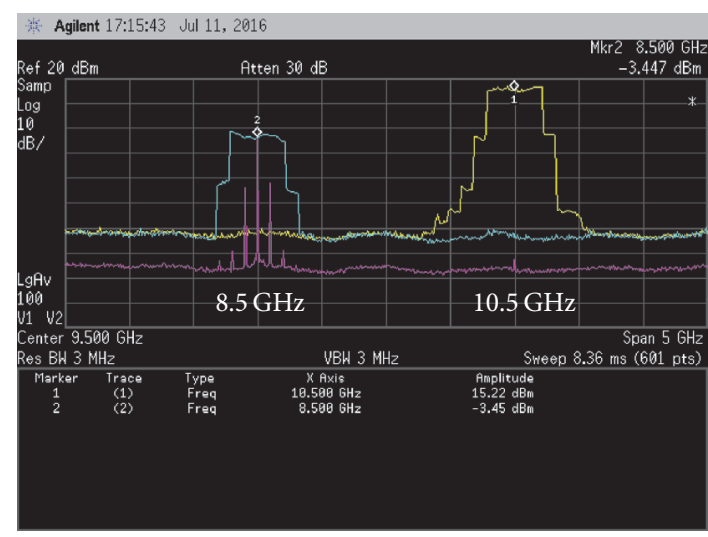

- TM

$\mathrm{BM}$

(b) Frequency converted spectrum of the tag module (TM)

FIGURE 5: Frequency spectra of the fabricated system.

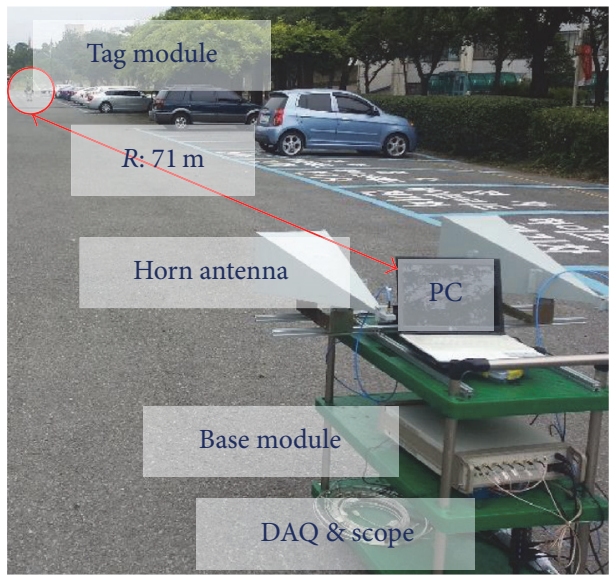

(a) Base module

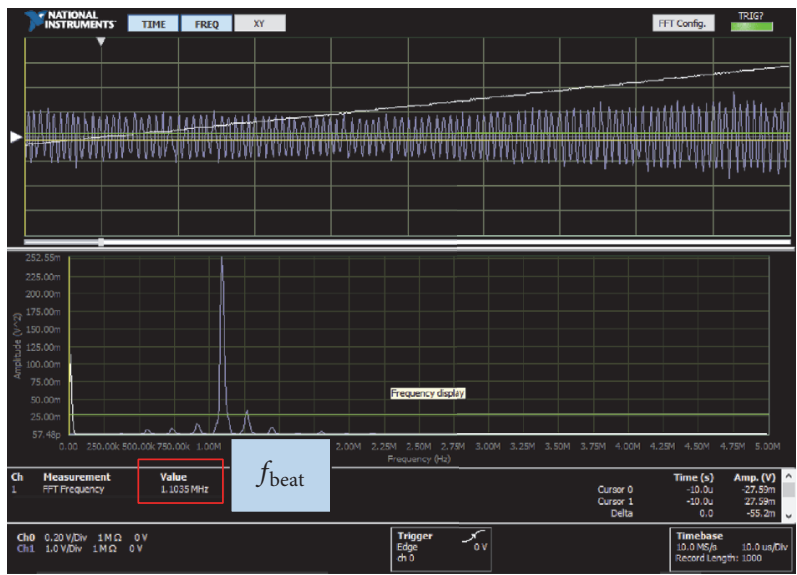

(c) NI-SCOPE GUI for $f_{\text {beat }}$

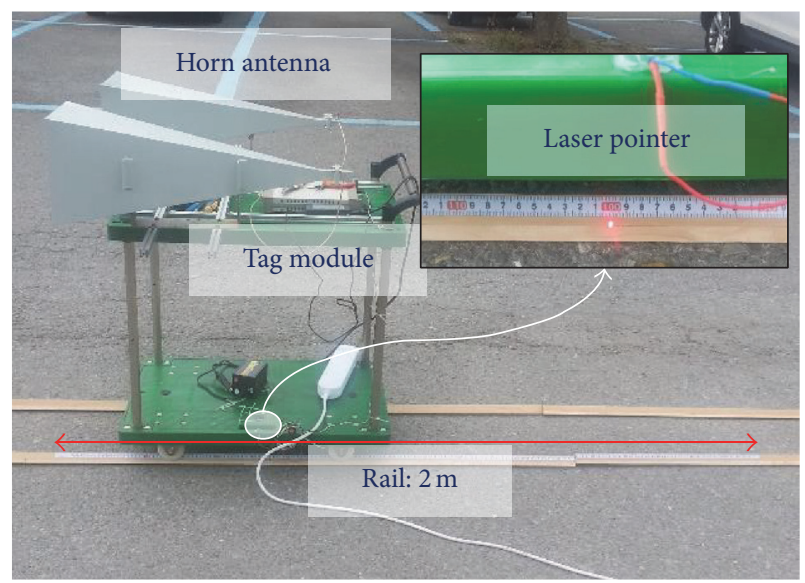

(b) Tag module

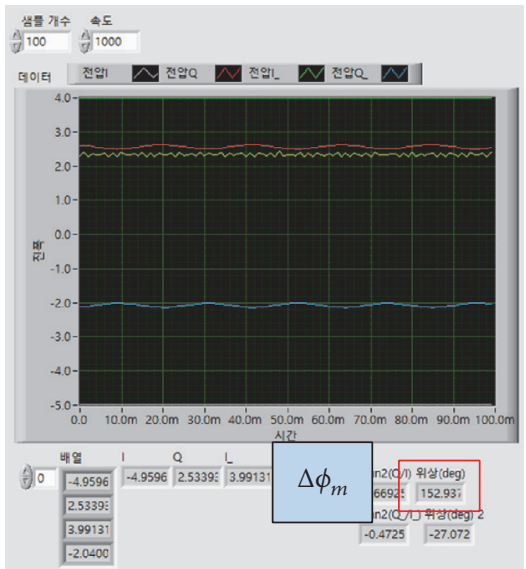

(d) LabVIEW program for $\Delta \phi_{m}$

FIgURE 6: Outdoor measurement setup. 


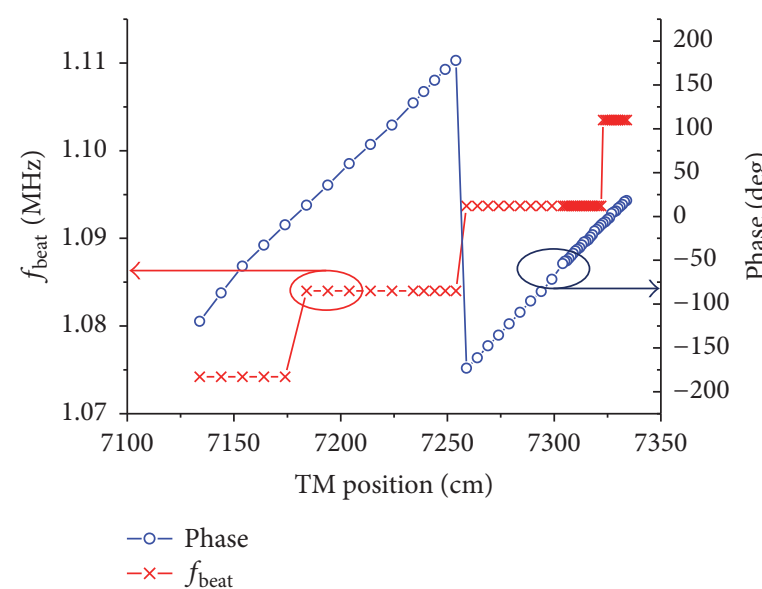

(a) Measured beat frequency $\left(f_{b}\right)$ and phase delay $(\Delta \phi)$

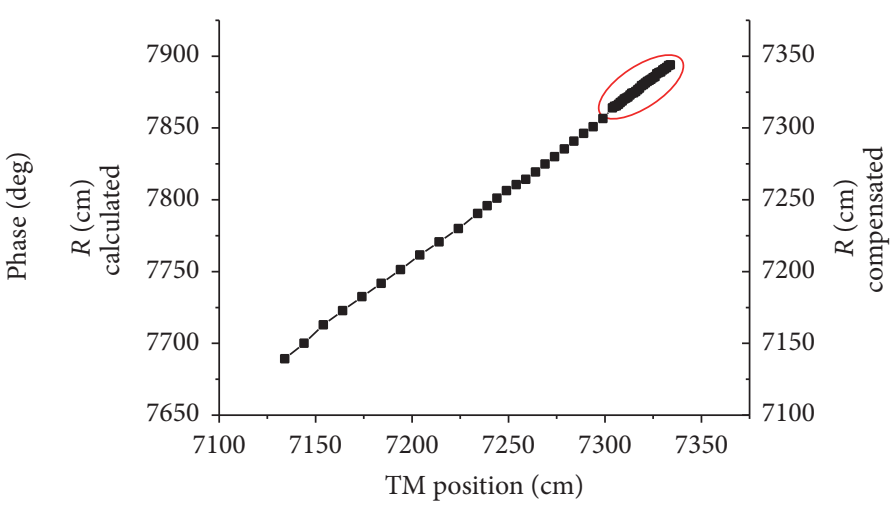

(b) Calculated distance $(R)$ using $f_{b}$ and $\Delta \phi$

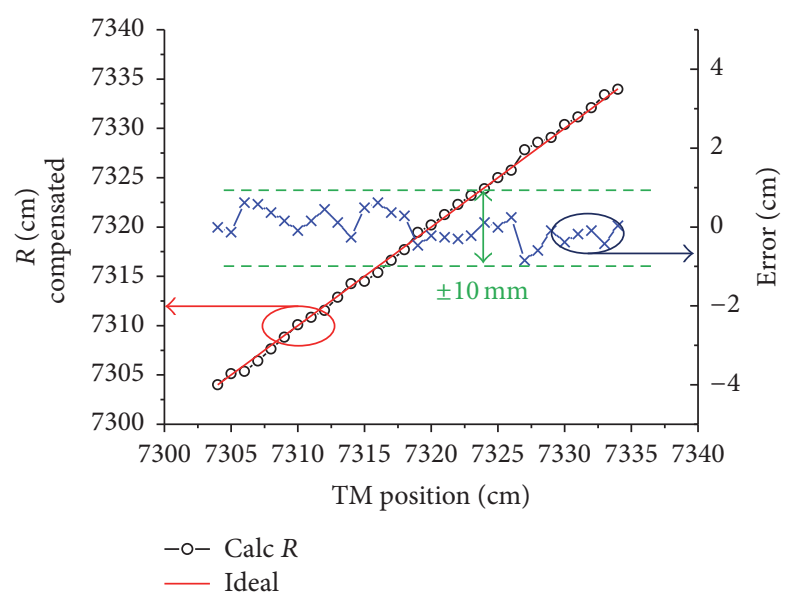

(c) Zoomed up portion of Figure 7(b) and the distance error

FIGURE 7: Measurement results of proposed system in outdoor fields.

TABLE 3: Comparison of high-accuracy radar systems.

\begin{tabular}{|c|c|c|c|c|c|}
\hline & System architecture & Center frequency & Bandwidth & Error & Operating range \\
\hline This work & $A M-F M$ & $10.5 / 8.5 \mathrm{GHz}$ & $620 \mathrm{MHz}$ & $10 \mathrm{~mm}$ & $70 m$ \\
\hline [3] & UWB & $5.4 \sim 10.6 \mathrm{GHz}$ & $5200 \mathrm{MHz}$ & $5 \mathrm{~mm}$ & $5 \mathrm{~m}$ \\
\hline [4] & UWB & 7 8 GHz & $1000 \mathrm{MHz}$ & $1.7 \mathrm{~cm}$ & $10 \mathrm{~m}$ \\
\hline [5] & UWB & $3.7 \sim 5 \mathrm{GHz}$ & $1300 \mathrm{MHz}$ & $20 \mathrm{~cm}$ & $8 \mathrm{~m}$ \\
\hline [6] & UWB & $3.2 \sim 5.2 \mathrm{GHz}$ & $2000 \mathrm{MHz}$ & $1 \mathrm{~cm}$ & $8 \mathrm{~m}$ \\
\hline [7] & UWB & $0.01 \sim 5 \mathrm{GHz}$ & $4990 \mathrm{MHz}$ & $1.5 \mathrm{~cm}$ & $2 \mathrm{~m}$ \\
\hline [8] & FMCW & $5.8 \mathrm{GHz}$ & - & $10 \mathrm{~cm}$ & $40 \mathrm{~m}$ \\
\hline [9] & FMCW & $5.8 \mathrm{GHz}$ & $150 \mathrm{MHz}$ & $18 \mathrm{~cm}$ & $40 \mathrm{~m}$ \\
\hline
\end{tabular}

\section{Competing Interests}

The authors declare that they have no competing interests.

\section{Acknowledgments}

This research was supported by the National R\&D Program through the National Research Foundation of Korea (NRF) funded by the Ministry of Education, Science, and Technology (NRF-2015M1A7A1A02002291).

\section{References}

[1] S. Tokoro, "Automotive application systems of a millimeterwave radar," in Proceedings of the IEEE Intelligent Vehicles Symposium, pp. 260-265, Tokyo, Japan, September 1996.

[2] T. Bao, J. Wang, and Y. Yao, "A fiber optic sensor for detecting and monitoring cracks in concrete structures," Science China Technological Sciences, vol. 53, no. 11, pp. 3045-3050, 2010.

[3] C. Zhang, M. J. Kuhn, B. C. Merkl, A. E. Fathy, and M. R. Mahfouz, "Real-time noncoherent UWB positioning radar 
with millimeter range accuracy: theory and experiment," IEEE Transactions on Microwave Theory and Techniques, vol. 58, no. 1, pp. 9-20, 2010.

[4] B. Waldmann, R. Weigel, and P. Gulden, "Method for high precision local positioning radar using an ultra wideband technique," in Proceedings of the IEEE MTT-S International Microwave Symposium Digest (MTT '08), pp. 117-120, IEEE, Atlanta, Ga, USA, June 2008.

[5] A. Fujii, H. Sekiguchi, M. Asai, S. Kurashima, H. Ochiai, and R. Kohno, "Impulse radio UWB positioning system," in Proceedings of the IEEE Radio and Wireless Symposium (RWS '07), pp. 55-58, Long Beach, Calif, USA, January 2007.

[6] Z. N. Low, J. H. Cheong, C. L. Law, W. T. Ng, and Y. J. Lee, "Pulse detection algorithm for line-of-sight (LOS) UWB ranging applications," IEEE Antennas and Wireless Propagation Letters, vol. 4, no. 1, pp. 63-67, 2005.

[7] R. Zetik, J. Sachs, and R. Thomä, "UWB localization-active and passive approach," in Proceedings of the 21st IEEE Instrumentation and Measurement Technology Conference (IMTC '04), vol. 2, pp. 1005-1009, May 2004.

[8] A. Stelzer, K. Pourvoyeur, and A. Fischer, "Concept and application of LPM-a novel 3-D local position measurement system," IEEE Transactions on Microwave Theory and Techniques, vol. 52, no. 12, pp. 2664-2669, 2004.

[9] F. Ellinger, R. Eickhoff, R. Gierlich et al., "Local positioning for wireless sensor networks," in Proceedings of the IEEE Globecom Workshops, pp. 1-6, Washington, DC, USA, November 2007.

[10] G. M. Brooker, "Understanding millimetre wave FMCW radars," in Proceedings of the 1st International Conference on Sensing Technology, pp. 152-157, IEEE, Palmerston North, New Zealand, November 2005.

[11] B. R. Mahafza, Radar Systems Analysis and Design Using MATLAB, Chapman and Hall/CRC, Boca Raton, Fla, USA, 2000.

[12] G. R. Curry, Radar System Performance Modeling, Artech House, 2nd edition, 2004.

[13] E. Hyun and J. H. Lee, "Method to improve range and velocity error using de-interleaving and frequency interpolation for automotive FMCW radars," International Journal of Signal Processing, Image Processing and Pattern Recognition, vol. 2, no. 2, 2009.

[14] O. K. Nilsen and W. D. Boyer, "Amplitude modulated CW radar," IRE Transactions on Aerospace and Navigational Electronics, vol. 9, no. 4, pp. 250-254, 1962.

[15] C. Laviron, A. J. H. Donné, M. E. Manso, and J. Sanchez, "Reflectometry techniques for density profile measurements on fusion plasmas," Plasma Physics and Controlled Fusion, vol. 38, no. 7, pp. 905-936, 1996.

[16] D. R. Brunfeldt and F. T. Ulaby, "Active reflector for radar calibration," IEEE Transactions on Geoscience and Remote Sensing, vol. 22, no. 2, pp. 165-169, 1984. 


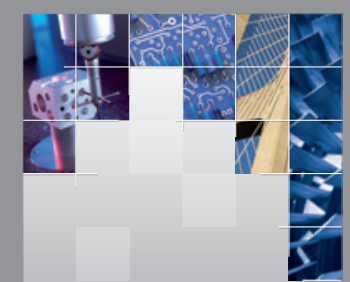

\section{Enfincering}
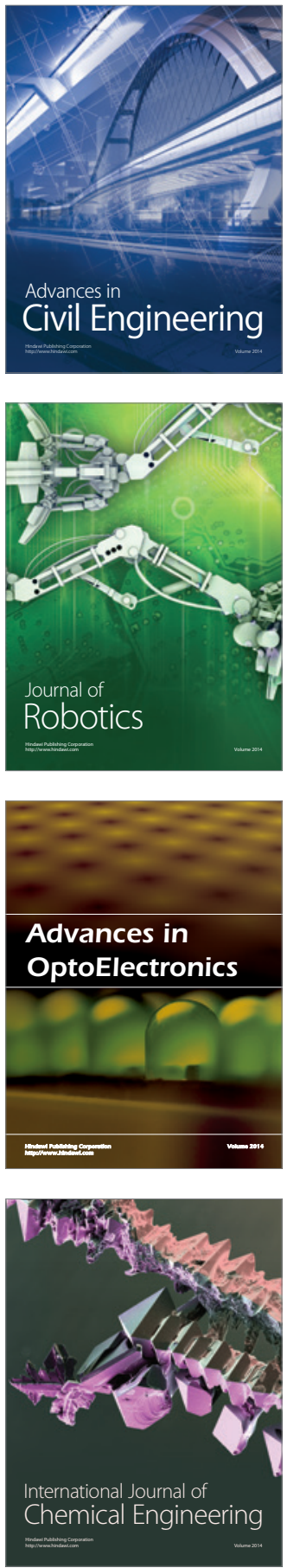

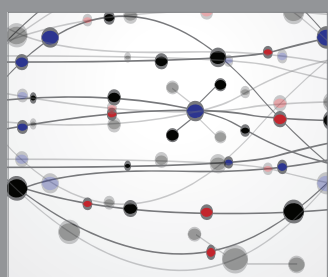

The Scientific World Journal

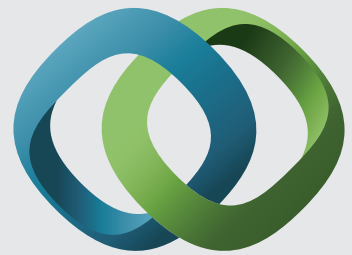

\section{Hindawi}

Submit your manuscripts at

https://www.hindawi.com
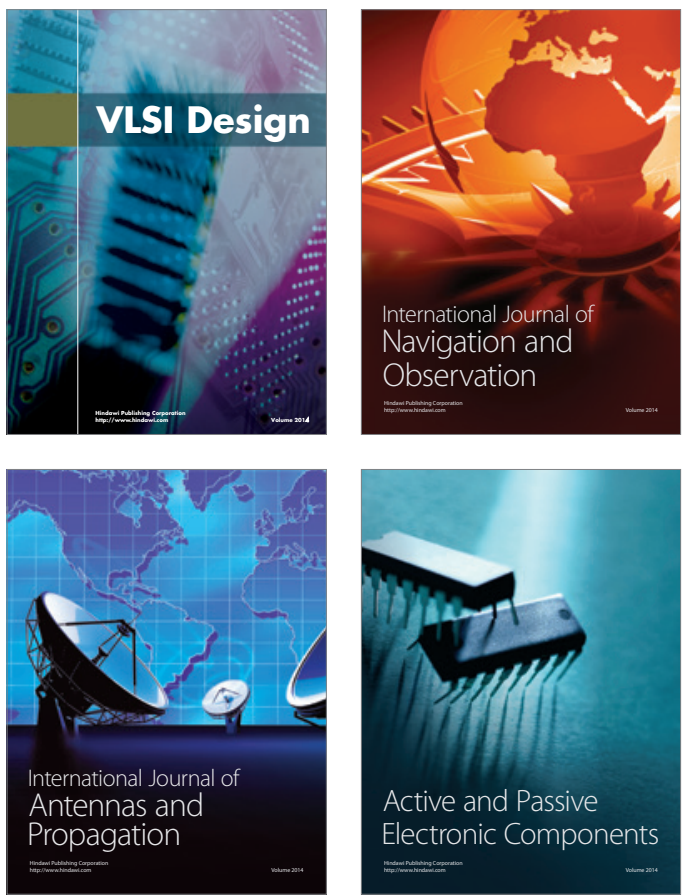
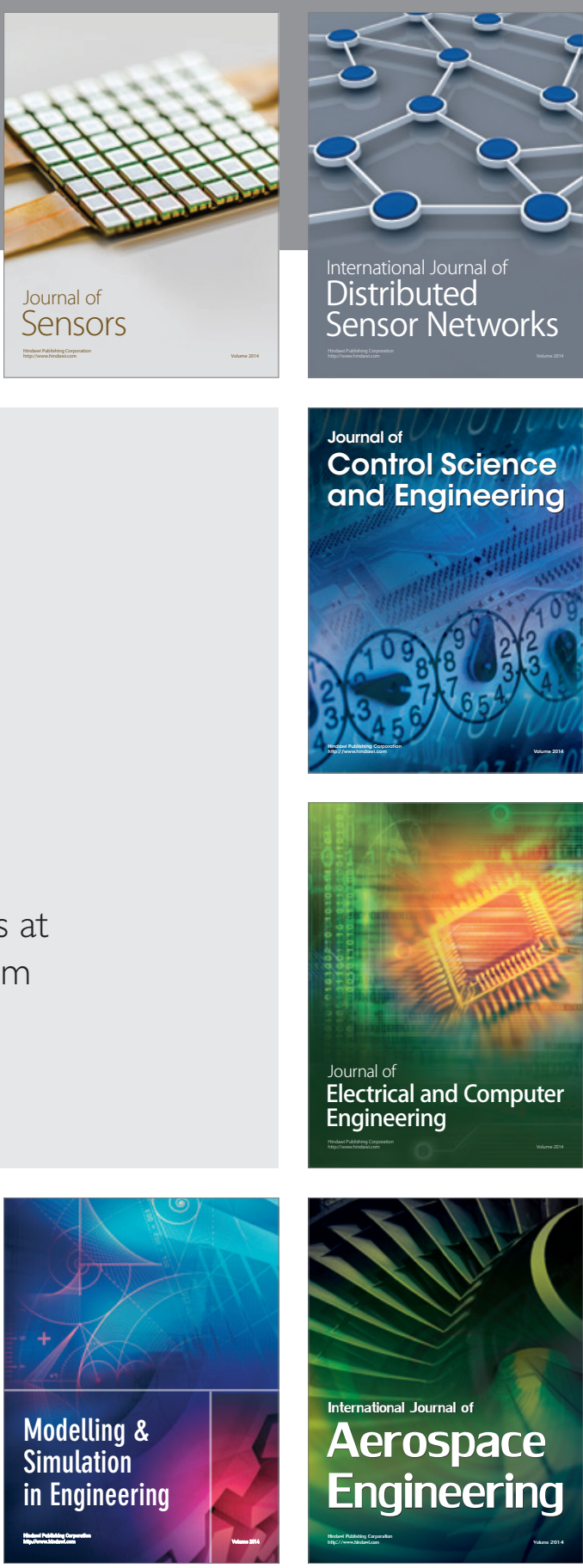

International Journal of

Distributed

Sensor Networks

$-$

Joumal of

Control Science

and Engineering
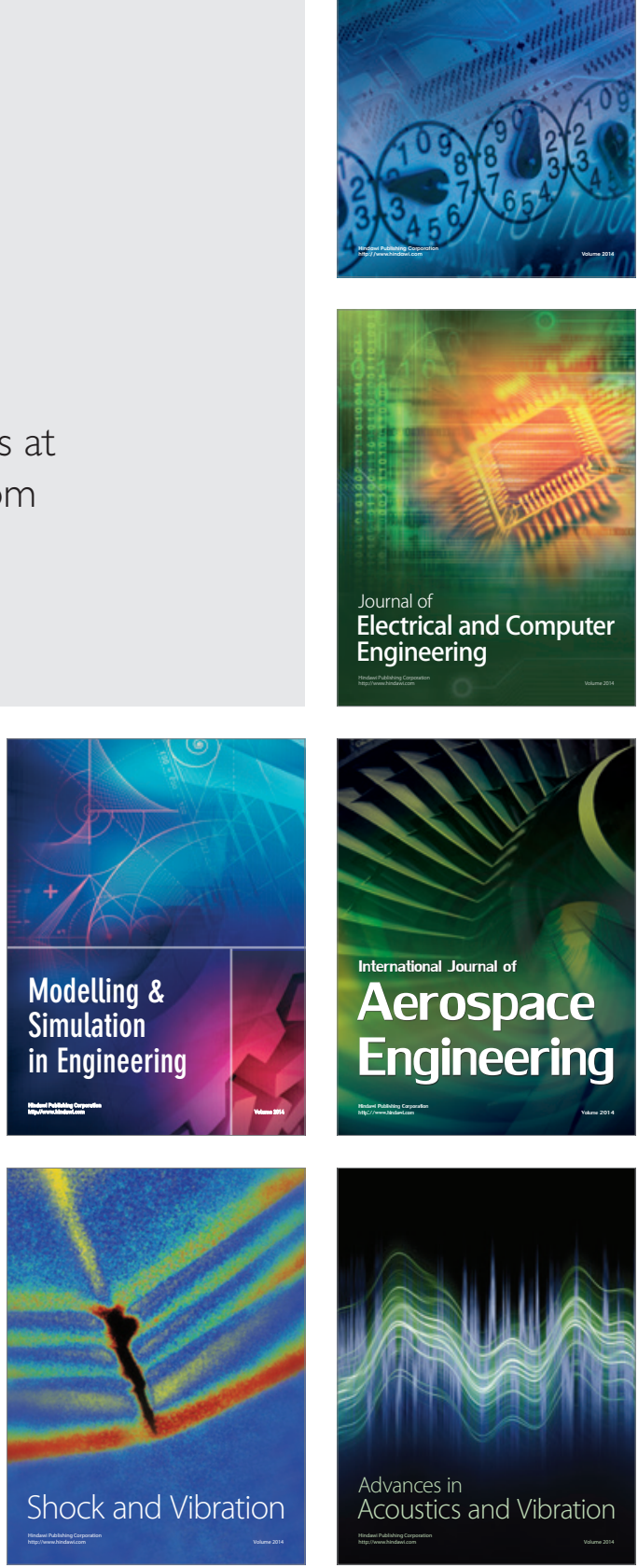